\title{
Quarterly NAFLD therapy and monitoring disease progression
}

\author{
Pankaj Puri, Vijayalakshmi Aravindan Arun
}

ABSTRACT

Department of Internal Medicine, Armed Forces Medical College, Pune.

Correspondence: Pankaj Puri

Email:puripankaj@gmail.com
Non-alcoholic fatty liver disease (NAFLD) is a major cause of chronic liver disease globally. The spectrum of NAFLD ranges from simple steatosis to non-alcoholic steatohepatitis (NASH) which can progress to advanced fibrosis, cirrhosis and hepatocellular carcinoma. NAFLD is associated with metabolic syndrome, type 2 diabetes mellitus and increased cardiovascular disease (CVD) which contribute to the morbidity and mortality besides that due to hepatic disease. While simple steatosis is relatively benign, NASH is associated with increased mortality. Hence differentiating between these two and quantification of fibrosis are necessary for deciding on therapy and monitoring of progression of the disease. Life style measures, weight loss, diet and exercise remain the cornerstone of management of NAFLD. While there is a significant amount of ongoing research in pharmacological agents for management of NAFLD, there are limited amount of agents with proven efficacy.

KEYWORDS: NAFLD; non-alcoholic fatty liver disease; NASH, nonalcoholic steatohepatitis.

Abbreviations used: AASLD: American Association for the Study of Liver Diseases; ALT: alanine aminotransferase; anti-HCV: antibody for hepatitis C; ARFI: acoustic radiation force impulse imaging; AST: aspartate aminotransferase; ALP: alkaline phosphatase; BMI: body mass index; CAP: controlled attenuation parameter; CVD: cardiovascular diseases; EASL: European Association for the Study of the Liver; EASD: European Association for the Study of Diabetes; EASO: European Association for the Study of Obesity; ELF: enhanced liver fibrosis; FFA: free fatty acids; FXR: Farnesoid X receptor; GLP: glucagon-like-peptide; GGT: gammaglutamyltranspeptidase; HBsAg: hepatitis B surface antigen; HCC: hepatocellular carcinoma; IR: insulin resistance; LT: liver transplantation; NAFLD: non-alcoholic fatty liver disease; NAFL: non-alcoholic fatty liver; NASH: nonalcoholic steatohepatitis; NAS: NAFLD activity score; NCEP ATP:National Cholesterol Education Programme Adult Treatment Panel; NICE: National Institute of Health and Care Excellence; OCA: Obeticholic acid; PPAR: peroxisomal proliferator-activated receptors; PUFA: polyunsaturated fatty acids; SAF: Steatosis Activity Fibrosis; TCI: total calorie intake; TE: Transient elastography; T2DM: type 2 diabetes mellitus; TG: triglycerides; US: Ultrasound. 


\section{Introduction}

Non-alcoholic fatty liver disease (NAFLD) is defined as excess accumulation of fat in the liver, which cannot be attributed to causes such as alcohol intake or drugs. The spectrum of NAFLD ranges from simple steatosis or non alcoholic steatosis (NAFL) to non-alcoholic steatohepatitis (NASH), cirrhosis, and hepatocellular carcinoma (HCC). ${ }^{1}$ Hepatic steatosis is considered pathological when it affects $>5 \%$ of the hepatocytes. ${ }^{2}$ Patients with NAFL have the presence of fat in the liver, with or without the presence of mild lobular inflammation. NASH is defined as hepatic steatosis and inflammation associated with the presence of hepatocyte injury (evident as hepatocyte ballooning), with or without the presence of hepatic fibrosis. ${ }^{3}$ These definitions are summarized in table $\mathbf{1}{ }^{4}$

NAFLD is the most common cause of chronic liver disease worldwide. It is expected that NASH related cirrhosis and NASH related HCC may soon become the most frequent indications for liver transplantation. ${ }^{3}$ It is estimated that NAFLD affects $30 \%$ of the general population who have radiological evidence of steatosis and $8 \%$ of them have raised transaminases due to NASH, although many of them go unrecognized. ${ }^{5-9}$ Studies from India show that the prevalence of NAFLD varies from $9 \%$ to $35 \%$ and the prevalence is lower in the rural population. ${ }^{10}$ Extrapolation of Indian data suggests that there may be at least 25 million patients with NAFLD in India. ${ }^{11}$

NAFLD is a multisystem disease. It is not only a marker of metabolic syndrome, but also a risk factor for cardiovascular diseases (CVD), chronic kidney disease and type 2 diabetes mellitus (T2DM). Moreover, it is a risk factor for malignancy (HCC, colorectal carcinoma) and other conditions (sleep apnea, osteoporosis, psoriasis, and polycystic ovary syndrome). ${ }^{12}$ Besides the liverrelated mortality, patients with NAFLD have an excess mortality due to associated conditions such as CVD. ${ }^{13,14}$ Hence, early therapeutic interventions in patients with NAFLD may have benefits beyond reducing the liver related morbidity and mortality.

\section{Pathogenesis of NAFLD and putative targets of therapy}

The pathogenesis of steatosis and NASH is unclearalthough development of insulin resistance (IR) is considered to play a central role. IR results in increased hepatic de novo lipogenesis, impaired inhibition of adipocyte tissue lipolysis, resulting in increased flux of fatty acids to the liver. IR also results in adipose tissue dysfunction with resulting altered production and secretion of adipokines and inflammatory cytokines. However, IR is not the sole factor responsible for NAFLD. Different theories of pathogenesis of NAFLD have been proposed. The 'twohit' hypothesis was initially proposed by Day and James. ${ }^{15}$ According to this hypothesis, the primary abnormality ('first hit') is accumulation of fat in the liver, most likely due to insulin resistance. Subsequently, a 'second hit' is caused by gut-derived endotoxins, oxidative stress or proinflammatory cytokines which induce hepatocyte injury and inflammation resulting in NASH.

However, it has been suggested that hepatic steatosis may result from several distinct injurious mechanisms. ${ }^{16}$ The 'two-hit' theory was therefore replaced by the 'multiple-hit' hypothesis in which, diverse parallel processes might act synergistically in genetically predisposed individuals resulting in the development of inflammation and progression of the disease. In the multiple-hit' theory, the first hit is insulin resistance which causes hyperinsulinaemia, resulting in an increased hepatic de novo lipogenesis and impaired inhibition

\section{Table 1: Definitions in the spectrum of non-alcoholic fatty liver disease}

\begin{tabular}{l|l} 
NAFLD & $\begin{array}{l}\text { Includes all forms of liver disease associated with hepatic steatosis in patients without significant alcohol } \\
\text { consumption, including simple steatosis, steatohepatitis and cirrhosis }\end{array}$ \\
\hline NAFL & Patients with hepatic steatosis without evidence of hepatic fibrosis or hepatocyte injury (hepatocyte ballooning) \\
\hline NASH & Patients with hepatic steatosis and inflammation with hepatocyte injury (ballooning) and/or hepatic fibrosis
\end{tabular}

NAFLD, Non-alcoholic fatty liver disease; NAFL, Non-alcoholic fatty liver; NASH, Non-alcoholic steatohepatitis. 
of adipose tissue lipolysis. This ultimately leads to an increased efflux of FFA from the adipose tissue to the liver. After the initial hepatic steatosis, the liver becomes vulnerable to hits from diverse parallel processes viz. oxidative stress, dysregulated hepatocyte apoptosis, dysbiosis of microbiota and changes in the adipokine levels leading to hepatocyte injury and progression from simple steatosis to NASH and fibrosis. ${ }^{17}$ The model suggests that inflammatory mediators derived from various tissues but especially from the gut and adipose tissue could play a central role in the cascade of inflammation, fibrosis, and finally tumour development. ${ }^{18}$

The hallmark of NAFLD is fat accumulation in the liver. Steatosis occurs whenever the synthesis or import of fat in the liver exceeds the export or degradation of fat. Fat accumulates in the liver in the form of triglycerides (TG). Triglycerides are synthesized from free fatty acids (FFA). There are three major sources of FFA in the liver: $60 \%$ from plasma FFAs (mostly from the adipose tissue), $25 \%$ from de novo lipogenesis and $15 \%$ from dietary fatty acids (FA) in the form of chylomicrons lipoproteins. ${ }^{19}$ The liver discards fat by oxidation or by exporting it as very-lowdensity lipoprotein. Alternatively, hepatocytes can shunt excess lipids to the synthesis of triglycerides and storage in lipid droplets. TG itself is not hepatotoxic. However, some of the other of lipids that accumulate in fatty livers (e.g., fatty acids, diacylglycerol, oxysterols, cholesterol, and phospholipids) can cause hepatocyte injury. ${ }^{20}$ These lipids can cause hepatocyte damage by mitochondrial damage, exacerbating the insulin resistance and hyperinsulinaemia, changes in cell signalling by interacting with transcription factors and innate immune receptors (toll-like receptors). This lipotoxicity results in endoplasmic reticulum stress, impaired autophagy and sterile inflammation that can potentiate cell injury and death. ${ }^{20}$

This lipotoxicity caused by lipids other than TG may initiate NASH. Under conditions of energy excess, adipose tissue produces adipocytokines that prevent adipocytes from assimilating FFAs and promote release of FFAs from adipose deposits. The increased FFAs delivered to the liver are converted to TG. The ability of TG synthesis to compensate for the increased FFA delivery to the liver may determine whether lipotoxicity will occur. NASH occurs because lipotoxic hepatocytes release factors that initiate wound-healing responses to the dying hepatocytes. ${ }^{20}$

Role of multiple processes acting in tandem is reflected by the lack of an effect of IR-reducing medications on NAFLD. Hence, treatment approaches beyond focusing on insulin resistance might be important. Based on the diverse processes hypothesised in the development of NASH, various potential treatment targets are being considered.

\section{Natural history of NAFLD}

The natural history and pathogenesis of NAFLD is not very clearly described. Majority of patients with NAFLD have simple steatosis which has a relatively benign prognosis. About $10 \%$ of patients have NASH which is associated with hepatocyte injury and inflammation and is the progressive form of NAFLD. ${ }^{21}$ Approximately 20-25\% with NASH will develop progressive hepatic fibrosis and progress to cirrhosis. ${ }^{22}$ In addition, patients with NASH are at risk of development of hepatocellular carcinoma without cirrhosis. ${ }^{23}$

Both the 'two-hit' theory and the 'multiple hit theory' are based on the assumption that NAFLD is a continuum from NAFL to NASH and that steatosis and insulin resistance precede the development of NASH. However, histological progression from simple steatosis to NASH is uncommon. ${ }^{24}$ It has been debated that simple steatosis and NASH appear as two distinct pathophysiological entities and progression from pure fatty liver to NASH appears to be so rare as to warrant publication. ${ }^{25}$ However, contrary to the current dogma, recent studies have shown that NAFL has the potential to progress to NASH with advanced fibrosis, particularly in patients who have or develop diabetes.

It is estimated that more than $40 \%$ of patients with NAFLD will have progressive fibrosis. A meta-analysis of studies of paired liver biopsy studies has shown that liver fibrosis progresses in patients with both NAFL and NASH. The rate of progression corresponds to 1 fibrosis stage every 14.3 years in NAFL and every 7.1 years in $\mathrm{NASH}$, and is doubled by arterial hypertension. ${ }^{26}$ 


\section{Management of NAFLD}

\section{Diagnosis}

Diagnosis of NAFLD requires the demonstration of steatosis on imaging and/or histology and exclusion of significant alcohol intake or other causes of liver disease and steatosis. ${ }^{27}$ Patients with NAFLD are usually asymptomatic at presentation. ${ }^{28}$ Clinical examination in patients without cirrhosis may reveal obesity, increased waist circumference, hypertension and hepatomegaly. Patients with NAFLD are usually diagnosed during evaluation of raised transaminases and/or gamma glutamyl transpeptidase (GGT) or during ultrasound evaluation of the abdomen. ${ }^{29}$ While the gold standard for diagnosis of NAFLD is liver biopsy, it is invasive and is associated with risks. Hence there is an increasing interest in validating non-invasive markers to validate the presence of steatosis and fibrosis.

\section{Clinical examination}

A detailed history should be taken including history of alcohol intake. In young women, changes in menstrual cycles and hirsutism may indicate polycystic ovarian syndrome, which may be associated with NAFLD. ${ }^{30}$ Physical examination should include body mass index, waist-to-hip ratio, blood pressure, stigmata of chronic liver disease and abdominal examination for hepatomegaly or ascites.

\section{Biochemical and serological investigations}

Blood samples should be done for (1) evaluating liver function tests [alanine aminotransferase (ALT), aspartate aminotransferase (AST), alkaline phosphatase (ALP), GGT, albumin, prothrombin time]; (2) assessing for associated metabolic abnormalities [lipid profile, blood sugar]; and (3) excluding alternative etiology of liver disease [hepatitis B surface antigen (HBsAg), antibody for hepatitis $\mathrm{C}$ (anti-HCV), autoimmune markers for autoimmune liver disease, serum ceruloplasmin, etc. as indicated].
Metabolic syndrome can be defined as per National Cholesterol Education Programme Adult Treatment Panel (NCEP ATP-III) criteria. ${ }^{31}$ According to these criteria, metabolic syndrome is diagnosed as presence of 3 or more of following 5 parameters: 1 . Waist circumference (WC) $>102 \mathrm{~cm}$ in men and $>88 \mathrm{~cm}$ in women, 2 . Hypertriglyceridemia: Triglycerides $>150 \mathrm{mg} / \mathrm{dL}$ or on specific medication, 3. Low HDL-C: $<40 \mathrm{mg} / \mathrm{dL}$ and $<50 \mathrm{mg} / \mathrm{dL}$, for men and women respectively, or specific medication, 4. Hypertension: $\mathrm{BP}>130 \mathrm{~mm}$ systolic or $>$ $85 \mathrm{~mm}$ diastolic or specific medication, 5. Fasting plasma glucose $>100 \mathrm{mg} / \mathrm{dL}$ or specific medication or previously diagnosed Type 2 DM. However, in South Asians, T2DM, dyslipidemia and cardiovascular morbidity occur at lower body weight. The proposed cut-offs for measures of obesity in South Asians are lower (BMI 23-24.9 kg/ $\mathrm{m} 2$ for overweight and $\geq 25 \mathrm{~kg} / \mathrm{m} 2$ for obesity, waist circumference $\geq 80 \mathrm{~cm}$ for women and $\geq 90 \mathrm{~cm}$ for men for abdominal obesity). ${ }^{32}$

\section{Imaging for detection of steatosis}

Ultrasound (US) is a most commonly employed method for detection of hepatic steatosis. It is cost-effective and can be used for screening the population at risk. However, it is operator dependant and may not be accurate in detecting mild steatosis. Steatosis cannot be reliably detected by US when the steatosis is less than $30 \% .{ }^{33,34}$ Computed tomography and magnetic resonance imaging are more sensitive techniques for detection and quantification of fat. ${ }^{35}$ However, considering the exposure to radiation with computed tomography and the cost of the advanced modalities, US remains the first-line imaging technique for diagnosis of NAFLD.

Non-invasive methods for assessment of severity and monitoring of disease progression

Various non-invasive methods have been evaluated to identify advanced fibrosis in patients with NAFLD. These include the NAFLD fibrosis score, enhanced liver fibrosis (ELF) panel, fibrosis-4 (FIB-4) calculator, FibroTest, measurement of circulating levels of cytokeratin and transient elastography. NAFLD fibrosis score is 
calculated using a formula (http://nafldscore.com) which incorporates six variables (age, albumin, AST/ALT ratio, body mass index $[\mathrm{BMI}]$, hyperglycemia and platelet count). ${ }^{36}$ The plasma cytokeratin levels are increased in patients with NASH compared to simple steatosis with $66 \%$ sensitivity and $82 \%$ specificity. ${ }^{37}$ However it has limited utility as a single marker to identify NASH. The FIB-4 calculator estimates fibrosis using the patient's age, aminotransferase levels and platelet count. ${ }^{38}$ The FibroTest is another commercial panel of biochemical tests which diagnoses advanced fibrosis scores with moderate accuracy. ${ }^{39}$ The ELF panel is a panel of markers of three matrix turnover protein levels (hyaluronic acid, TIMP-1 and PIINP). ${ }^{40}$ The National Institute of Health and Care Excellence (NICE) have recommended that of all the available non-invasive tests for identifying the NAFLD patients with advanced fibrosis, ELF was found to be the most cost effective in identifying patients with advanced fibrosis. In people incidentally detected to have NAFLD, advanced fibrosis is diagnosed if they have an ELF score of $\geq 10.51 .^{41}$

Transient elastography (TE) by FibroScan is widely used to measure liver stiffness, which is a surrogate marker for hepatic fibrosis. Simultaneous measurement of steatosis can also be carried out with FibroScan, using the controlled attenuation parameter (CAP) technique. ${ }^{42}$

However, there are limitations in using TE in patients with NAFLD. FibroScan may not be easy to perform in obese patients. The results may be invalid in older patients, patients with central obesity or T2DM. ${ }^{43} \mathrm{~A}$ Fibroscan XL probe is available for use in obese people to decrease failures in liver stiffness measurement. However, even with an XL probe, $10 \%$ obese patients have a difference of $\geq 2$ fibrosis stages between $\mathrm{TE}$ and liver biopsy. ${ }^{44}$ In addition to TE, two other modalities which have shown promising results include acoustic radiation force impulse imaging (ARFI) and MRI elastography.

\section{Liver biopsy}

While liver biopsy is the gold standard for identifying NASH and advanced fibrosis, it is not without its risks. Liver biopsy should be considered in patients with suspected NAFLD when there is diagnostic uncertainty of competing causes of liver steatosis or a co-existing liver disease which cannot be excluded without a liver biopsy. Liver biopsy shows macrovesicular steatosis in NAFLD though patchy microvescicular steatosis may be seen in $10 \%$ cases of NAFLD. Steatosis may be zone 3 (pericentral) or panacinar and may become irregularly distributed or vanish when cirrhosis develops. In paediatric NAFLD, steatosis may be seen in zone 1 . The histological features of NASH include ballooned hepatocytes, lobular inflammation and fibrosis. The other features seen in NASH include apoptotic bodies and Mallory-Denk bodies. Various scoring systems have been used to assess disease severity. These include NAFLD activity score (NAS) and the Steatosis Activity Fibrosis (SAF) score though these are usually used in clinical trials. ${ }^{45}$

\section{Treatment}

The European Association for the Study of the Liver (EASL), European Association for the Study of Diabetes (EASD) and European Association for the Study of Obesity (EASO) have recently jointly issued the EASL-EASD-EASO clinical practice guidelines for the diagnosis, management and follow up of NAFLD patients. ${ }^{46}$ The National Institute of Health and Care Excellence (NICE) has also issued recent guidelines for the diagnosis and management of NAFLD. ${ }^{41}$ A systematic review has evaluated ten recently published guidelines for clinical management of NAFLD and had suggested an algorithm for diagnosis and management of NAFLD based on the evaluation of these guidelines. ${ }^{47}$

\section{Weight reduction interventions}

Numerous studies have shown the benefits of weight loss in NAFLD. ${ }^{48-50}$ Weight reduction is recommended in the management of patients with NAFLD who are overweight or obese. The first step in this direction is lifestyle measures which include dietary change, physical activity and behavioural therapy. However, while weight reduction is recommended in the treatment of NAFLD, achieving weight loss and maintaining it remains a 
challenge in overweight and obese persons. The other modalities used for inducing weight loss include the use of pharmacological agents and bariatric surgery.

\section{Goals of weight loss}

Weight loss of $3-5 \%$ of body weight is associated with improvement in hepatic steatosis. However, resolution of necroinflammation has been correlated with weight loss of $>7 \% .{ }^{48}$ In a prospective study of 293 patients with $\mathrm{NASH}$, Vilar-Gomez et $\mathrm{al}^{50}$ showed that in patients who had $\geq 10 \%$ weight loss, $90 \%$ had resolution of NASH and $45 \%$ had resolution of fibrosis. A similar regression in hepatic fibrosis has been shown by other workers in patients who achieve weigh loss of $\geq 10 \%{ }^{51}$ It is recommended that weight loss of at least $7 \%$ is necessary to improve hepatic inflammation and a greater weight loss of $\geq 10 \%$ of body weight is required for regression of hepatic fibrosis in NASH. ${ }^{52}$

\section{Life style measures}

Life style modifications including diet, physical activity and behavioural therapy are recommended as the first line therapy in the management of NAFLD. Behavioural therapy is the use of psychological techniques to modify maladaptive behaviour and has been shown to improve outcomes in diabetes and obesity. Barriers to lifestyle change should be addressed during consultation visits. ${ }^{53}$

Dietary recommendations should include calorie restriction and avoidance of foods which can promote NAFLD. Patients should be advised to reduce calories by $25 \%$ from the normal diet. ${ }^{5}$ Generally the calorie deficit should be by $500-1000 \mathrm{kcal} /$ day and the diet should provide at least $1200-1500 \mathrm{kcal} /$ day though this may require to be individualized and adjusted for weight loss. Carbohydrates should provide $45-60 \%$ of total calorie intake (TCI) and should preferably not be refined. Total fats should account for $20-35 \%$ of TCI ( saturated fats 7 to $10 \%$ of TCI, polyunsaturated fats $5-10 \%$ of TCI and monounsaturated fats $15-20 \%$ of TCI, trans-fatty acids $<1 \%$ of TCI). Proteins should comprise $20 \%$ of TCI. ${ }^{54}$ Foods which should be excluded from the diet include processed food, and foods and beverages high in added fructose. ${ }^{46}$
There is currently no universal consensus on the type of diet that NAFLD patients should follow. Lowcarbohydrate and Mediterranean diet are effective alternatives to low-fat diet. The Mediterranean diet is abundant in cereals, vegetables and legumes. The Mediterranean diet contains predominantly monounsaturated fats from olive oil with a greater ratio of omega-3 polyunsaturated fatty acids (PUFAs) to omega-6 PUFAs, whereas the ratio is reversed in Western diets. ${ }^{55}$ In a study comparing Mediterranean diet with a low-carbohydrate diets or a low-fat diet, Shai et $\mathrm{al}^{56}$ found that the mean weight loss was similar in the lowcarbohydrate and Mediterranean diet cohorts and that both were significantly better than the low-fat group. The low-carbohydrate group showed better more beneficial effects on lipid profiles while glycemic control was better in the Mediterranean diet group.

Alcohol intake should be kept below the threshold limit of $30 \mathrm{gm} /$ day for men and $20 \mathrm{gm} /$ day for women.

\section{Anti-obesity drugs}

The medicines prescribed for weight loss include: sibutramine, orlistat and rimonabant. There have been safety concerns about these medications prescribed for obesity. Sibutramine has been associated with risk of adverse cardiovascular event; orlistat may be associated with liver injury and rarely acute liver failure; and rimonabant has been associated with psychiatric side effects. $^{57}$

Besides the above drugs, another drug which may be effective in the treatment of obesity in patients with NAFLD is liraglutide, a glucagon-like-peptide (GLP)1 receptor agonist used in treatment of type 2 diabetes mellitus. Liraglutide is an anorectic and has been shown to be effective in promoting weight loss. ${ }^{58,59}$

\section{Exercise}

Exercise may improve insulin sensitivity and hepatic steatosis, regardless of weight loss. ${ }^{60}$

All patients should be advised to carry out of 150300 minutes of moderately-intense physical exercise per week in 3-5 sessions. ${ }^{46,57}$ The volume of exercise is an 
important factor and it has been shown that 250 minutes of weekly exercise was more effective than 150 minutes. ${ }^{61}$

Besides aerobic training, resistance training (which improves muscle strength through repetitive weight-based exercises), also improves NAFLD. ${ }^{62,63}$ A combination of aerobic training and resistance training may give the maximum benefit though further studies are required to confirm the same. ${ }^{53}$

\section{Bariatric surgery}

Bariatric surgery is recommended in severely obese patients who are unable to lose weight by lifestyle measures. Bariatric surgery has been shown to be effective in improving both the biochemical and histological features of NAFLD. ${ }^{64}$ Gastric bypass may be more effective than gastric banding in improving liver disease, possibly due to greater weight loss and a rapid delivery of nutrients to ileum thereby increasing release of hormones which increase satiety. ${ }^{65,66}$

In a Cochrane review, four studies showed worsening of fibrosis in patients with NAFLD, mainly in patients with advanced fibrosis, suggesting that the surgery may not be safe in all patients. ${ }^{67}$ Considering the risk of worsening of fibrosis, patients undergoing bariatric surgery should be closely monitored.

Currently, it is unclear whether bariatric surgery should be done primarily for the treatment of NASH and more studies are required to determine which type of bariatric surgery may confer benefit in patients with NASH.

\section{Pharmacotherapy}

Drug therapies for NAFLD are targeted at the other metabolic syndrome-associated illness such as obesity, type 2 diabetes mellitus, hypertension and hyperlipidemia besides the management of hepatic disease. The understanding of the mechanisms in the development of NAFLD have resulted in identification of potential targets of pharmacological interventions aimed at decreasing the inflammation and reversing the fibrosis in patients with NAFLD. The drugs being evaluated in the in the management of NAFLD are Insulin sensitizing agents, medication targeting oxidative stress, medication which decreases inflammation and cell death, medication targeting the lipid metabolism, medication which is targeted at the interaction between the gut and the liver and antifibrotic agents which prevent or reduce fibrosis. The indications for drug therapy in NAFLD include advanced fibrosis, high necroinflammatory activity or early stage NASH with increased risk of progressive fibrosis (Age > 50 years, diabetes, metabolic syndrome, increased alanine aminotransferase). ${ }^{46}$

\section{(1) Insulin-sensitizing agents}

Insulin resistance is considered to be central to the pathogenesis and progression of NAFLD. Insulin sensitizing agents are thought to improve the hepatic histology and transminase levels by improving insulin levels and thereby the steatosis.

\section{(a) Metformin}

Metformin was the first insulin-sensitizing agent tested in patients with NAFLD. Metformin may reduce insulin resistance and liver enzyme activities but results from trials on its effects on hepatic histology in NAFLD have been controversial. ${ }^{68}$

While initial studies had shown the benefits of metformin, ${ }^{69-71}$ subsequent studies have failed to show any effect on liver histology. A meta-analysis has shown that metformin was not effective in patients with NAFLD. ${ }^{72}$ Metformin is not recommended for use in NAFLD by the current guidelines. ${ }^{46}$ There is some data of the role of metformin in preventing HCC. ${ }^{73,74}$ However this is too preliminary to justify its use for this role.

\section{(b) Peroxisome Proliferator-Activated Receptors (PPAR) Agonists}

The peroxisomal proliferator-activated receptors (PPAR) are a family of nuclear receptors that bind fatty acids and their derivatives. PPAR- $\alpha$ receptors are highly expressed in the liver, kidney and muscle, PPAR $-\gamma$ receptors are mainly expressed in the adipose tissue and PPAR- $\beta / \delta$ receptors are universally expressed. ${ }^{75}$ 
PPAR- $\gamma$ receptors, which are expressed on the adipose tissue, are involved in glucose metabolism and control of lipogenesis. The thiazolidinedione pioglitazone is a PPAR gamma agonists which has been evaluated in multiple trials for the treatment of NASH. ${ }^{76-79}$ The largest RCT of pioglitazone use in NASH is the PIVENS trial. This trial included 247 non-diabetic patients with biopsy proven NASH who were randomized to 96 weeks therapy with pioglitazone $30 \mathrm{mg}$, Vit E $800 \mathrm{IU}$ or placebo. Pioglitazone was associated with histological improvement in $34 \%$ of the patients compared with $19 \%$ of controls. Although this did not reach the pre-specified statistical significance regarding reduction in the NAS score, there was improvement in enzyme levels as well as histological improvement in steatosis and lobular inflammation. ${ }^{76}$ A meta-analysis of the use of pioglitazone in NASH has shown improvement of fibrosis with the use of the drug. ${ }^{80}$

However, there are concerns regarding the safety of long-term use of thioglitazones. Rosiglitazone has been associated with cardiac ischemia and heart failure. There are reports of increased bone fractures, congestive heart failure and bladder cancer with the use of Pioglitazone which have raised concerns about its long-term safety. ${ }^{81-83}$

Elafibranor is a dual PPAR $\alpha / Y$ receptor agonist which has been evaluated in the treatment of NASH. After exclusion of mild cases, high dose elafibranor $(120 \mathrm{mg} /$ day) was better than placebo in the resolution of NASH. ${ }^{84}$

Saroglitazar is a dual PPAR $\alpha / \gamma$ receptor agonist which is approved in India for the use in diabetic dyslipidemia. While available data of its benefit in NASH is limited, a double blind placebo controlled trial of saoglitazor in NASH is currently underway in India (Clinical Trials Registry-India CTRI/2015/10/006236) ${ }^{85}$

\section{(c) FarnesoidX receptor agonist (Obeticholic acid)}

Farnesoid X receptor (FXR) is a nuclear receptor which controls glucose and lipid metabolism and is expressed mainly in the liver and the gut. Obeticholicacid (OCA) is a FXR receptor agonist which decreases hepatic gluconeogenesis and improves insulin sensitivity.In the Farnesoid X Receptor Ligand Obeticholic Acid in NASH Treatment (FLINT) trial, 283 non-cirrhotic NASH patients were randomized to either receive OCA $25 \mathrm{mg}$ daily or placebo for a total of 72 weeks. The study showed that OCA was beneficial in improving NASH.However, pruritus and deranged lipid profile were the main side effects. $^{86}$

\section{(d) Glucagon-like peptide-1 (GLP-1)receptor} agonists

GLP-1 is produced by the intestinal cells in response to meal ingestion and acts on the pancreas to stimulate insulin secretion and inhibit glucagon secretion. It also enhances satiety, delays gastric emptying and decreases insulin resistance. ${ }^{87}$ GLP-1 receptor agonists like liraglutide and exenatide are used in treatment of diabetes. Unlike sulphonylureas, GLP-1 receptor agonists do not cause hypoglycaemia as they only promote insulin when there is a glucose trigger. The beneficial effect of GLP-1 liraglutide in histological resolution of NASH has been shown in the Liraglutide Efficacy and Action in Nonalcoholic Steatohepatitis (LEAN) study. ${ }^{88}$ However, more studies are required to see whether this is independent from the effect of weight loss alone.

\section{(2) Antioxidants and cytoprotective drugs}

Vitamin $\mathrm{E}$ has been shown to be beneficial in the treatment of non-diabetic patients with NASH in the PIVENS trial. ${ }^{76}$ However, in children, vitamin E failed to show a beneficial effect in improving NASH in the TONIC trial. ${ }^{89}$

While vitamin $\mathrm{E}$ is used for treatment of nondiabetic patients with NASH, the transaminase levels rises on discontinuation of the drug and vitamin $\mathrm{E}$ needs to be administrated for a long period of time. This long-term administration of vitamin $\mathrm{E}$ is associated with concerns of increased incidence of hemorrhagic stroke, prostatic cancer and mortality. ${ }^{90-92}$

Other anti-oxidants like ursodeoxycholic acid and silymarin have been tried but there is little evidence to support their use. Pentoxyphylline may decrease hepatic inflammation by decreasing tumour necrosis factor-alfa. While there is limited data to show its beneficial effect, the evidence to support its use is weak. ${ }^{93}$ 


\section{(3) Other drugs}

The use of $n-3$ polyunsaturated fatty acids has been suggested but studies have not shown significant histological improvement in NASH. ${ }^{94,95}$

Role of lipid-lowering drugs in NAFLD is inconclusive. While there is some data suggesting improvement in NASH with statins,${ }^{96}$ larger studies are needed to establish the effect of statins on liver histology. While pilot studies had shown the beneficial effects of ezetimibe in patients with NAFLD, ${ }^{97,98}$ a recent randomized controlled trial of 50 patients with NASH (the Magnetic Resonance Imaging and Elastography in Ezetimibe Versus Placebo for the Assessment of Treatment Response in NASH [MOZART] trial) showed that ezetimibe had no specific role in the treatment of NASH. ${ }^{99}$

While there are few available treatment options of proven efficacy in NAFLD, promising drugs are under development and under evaluation for regulatory approval. ${ }^{100}$

Therapeutic interventions which have been found to be effective in the management of NAFLD are shown in Table 2.

\section{Table 2 : Therapeutic interventions effective in NAFLD}

\begin{tabular}{|c|c|c|}
\hline STRATEGY & PUTATIVE MECHANISM OF ACTION & RESPONSE \\
\hline $\begin{array}{l}\text { DIET } \\
\text { Calorie deficit of } 500-1000 \mathrm{kcal} / \mathrm{d} \text {; } \\
\text { Carbohydrates: } 45-60 \% \mathrm{TCI} \text {; } \\
\text { Proteins : } 20 \% \mathrm{TCI} \\
\text { Fats : } 20-35 \% \text { TCI with saturated } \\
\text { fat }<10 \% \text { and Trans fats }<1 \%\end{array}$ & $\begin{array}{l}\text { Reduces insulin resistance and } \\
\text { cardiovascular risk; lipid profile reduces } \\
\text { oxidative stress }\end{array}$ & $\begin{array}{l}\text { First line of management } \\
>3 \%-5 \% \text { : reduces steatosis } \\
>7 \%-9 \% \text { : reduces necroinflammation } \\
>10 \% \text { : reduces fibrosis }\end{array}$ \\
\hline $\begin{array}{l}\text { EXERCISE } \\
150-300 \text { minutes of combined } \\
\text { moderate intensity aerobic exercise } \\
\text { (brisk walking) per week in } 3-5 \\
\text { sessions. Resistance training of at } \\
\text { least } 2 \text { sessions per week }\end{array}$ & $\begin{array}{l}\text { Improves insulin sensitivity, cardiovascular } \\
\text { health, overall mortality and cancer risk } \\
\text { independent of weight change }\end{array}$ & First line of management \\
\hline BARIATRIC SURGERY & $\begin{array}{l}\text { Induces sustained weight loss for up to } \\
\text { at least } 10 y \text { Reduces insulin resistance } \\
\text { andcardiovascular risk }\end{array}$ & $\begin{array}{l}\text { Reverses NASH in } 85 \% \text { of patients. } \\
\text { Reduces,necroinflammation,steatosisand } \\
\text { fibrosis; safety concerns about progression } \\
\text { of fibrosis postoperatively. }\end{array}$ \\
\hline $\begin{array}{l}\text { INSULIN SENSITISER: } \\
\text { PIOGLITAZONE }\end{array}$ & $\begin{array}{l}\text { PPAR } \text { yagonist, lipid } \\
\text { homeostasis, Inflammation, } \\
\text { and cell differentiation }\end{array}$ & $\begin{array}{l}\text { Reduces steatosis, necro-inflammation, } \\
\text { and fibrosis }\end{array}$ \\
\hline $\begin{array}{l}\text { ANTIOXIDANT: } \\
\text { VITAMIN E }\end{array}$ & anti-oxidative stress & $\begin{array}{l}\text { Reduces steatosis, necro-inflammation, } \\
\text { and fibrosis }\end{array}$ \\
\hline \multicolumn{3}{|l|}{ OTHER DRUGS } \\
\hline OBETICHOLIC ACID & $\begin{array}{l}\text { FXR agonist, increases energyhomeostasis } \\
\text { and lipidmetabolism; reduces } \\
\text { insulinresistance, inflammation, and } \\
\text { fibrotic pathways }\end{array}$ & $\begin{array}{l}\text { Phase III trials } \\
\text { Reduces steatosis, necro-inflammation, } \\
\text { and fibrosis }\end{array}$ \\
\hline LIRAGLUTIDE & $\begin{array}{l}\text { GLP1 agonist, promotes satiation,weight } \\
\text { loss, reduces insulinresistance }\end{array}$ & $\begin{array}{l}\text { Reducessteatosis, necroinflammation and } \\
\text { resolution of NASH }\end{array}$ \\
\hline PENTOXIFYLLINE & $\begin{array}{l}\text { Reduces inflammation (potentiallyby } \\
\text { inhibiting TNF), bloodviscosity, and platelet } \\
\text { aggregation }\end{array}$ & $\begin{array}{l}\text { Phase II completed Reduces steatosis and } \\
\text { necro-inflammation }\end{array}$ \\
\hline
\end{tabular}




\section{Liver transplantation for NAFLD}

Listing for liver transplantation (LT) in either NASH related decompensated cirrhosis or HCC should be done as per standard listing criteria. Patients with BMI $>40 \mathrm{~kg} /$ $\mathrm{m}^{2}$ are likely to have higher mortality after LT. In patients with compensated cirrhosis, it may be reasonable to attempt weight loss while patient is waiting for transplant. However in decompensated cirrhosis this may lead to protein calorie malnutrition. Patients with NASH should have appropriate risk stratification to exclude concomitant coronary artery disease. Intra-operatively, bariatric surgery may be considered at the time of LT in severe morbid obesity. For post-operative immunosuppression, steroid should either be withdrawn early or a steroid free regimen should be used. Glycaemic control will need to be optimised. For hyperlipidemia, pravastatin or ezetimibe are preferred drugs compared to atorvastatin or simvastatin as the latter may have interactions with calcineurin inhibitors. Post-operative monitoring of NASH patients after LT should be done to exclude disease recurrence. ${ }^{101}$

\section{Practical approach to management of patient with NAFLD}

Considering the limited available pharmacological agents which are effective in NASH and the concerns of the side effects of their long term use, therapy will need to be tailored depending not only on the stage and severity of the disease but also on the presence of co-morbid conditions like obesity or T2DM. Advice for treatment for patients with NASH and no or minimal fibrosis will be different than for that with stage IIIIV fibrosis.

The assessment for the presence of NASH and the stage of fibrosis is imperative prior to embarking on the treatment strategy. Patients should also be evaluated for all components of metabolic syndrome and for CVD.

A practical approach to management of patients with NAFLD is shown in Figure 1.

\section{Monitoring disease progression}

The progress of the disease is slow and depends on the presence or absence of NASH. A patient with only fatty liver would be expected to develop cirrhosis in about 57 years while a patient with NASH would develop it in 28 years and time would be halved by arterial hypertension. ${ }^{26}$ Patients with NASH who have fibrosis and hypertension should have close monitoring as they have a high risk of disease progression and development of cirrhosis. ${ }^{46} \mathrm{NAFL}$ is not associated with increased mortality ${ }^{102}$ probably as the

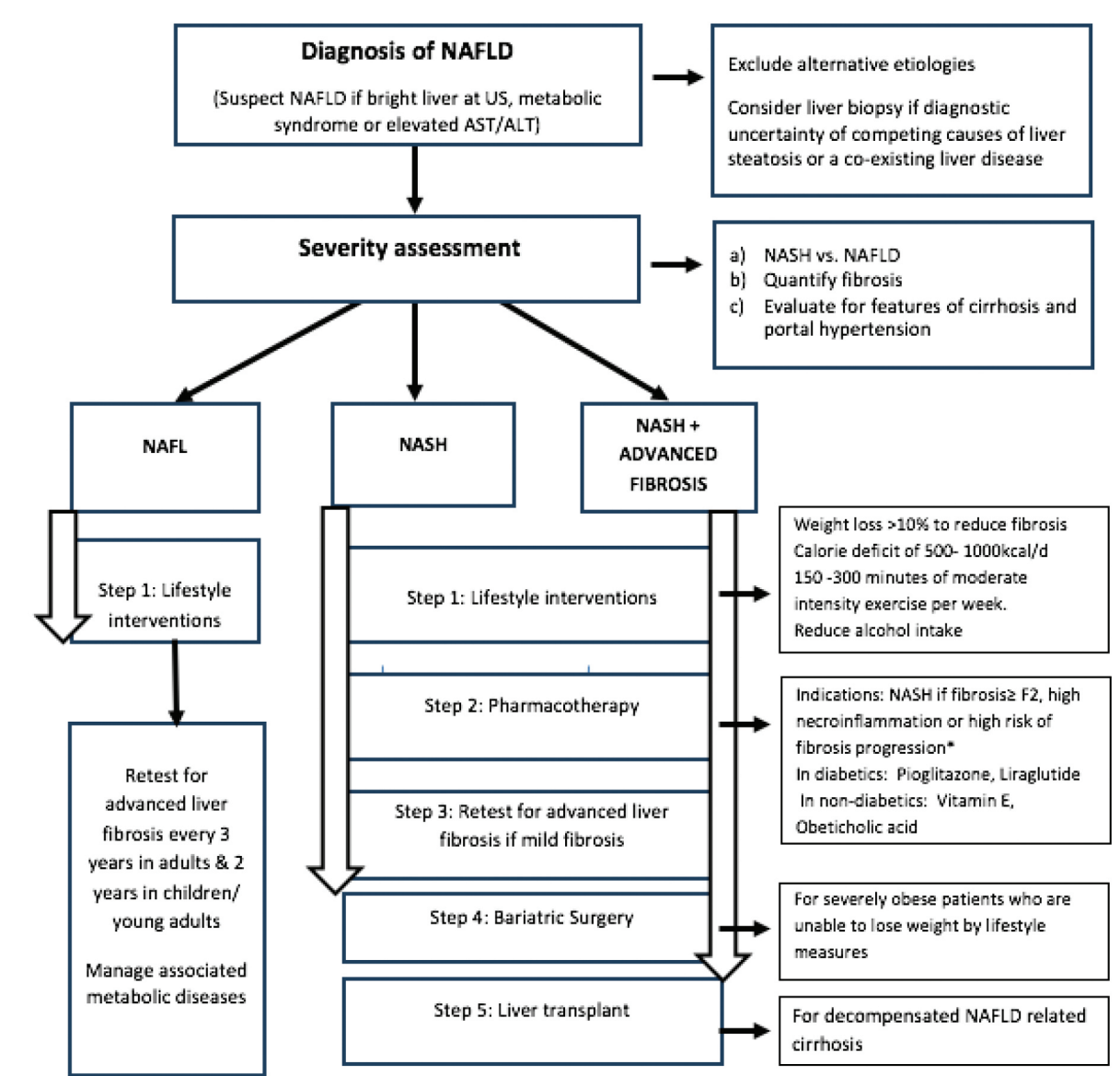

Figure 1: Clinical algorithm for the diagnosis and management of NAFLD. ALT, alanine aminotransferase, AST, aspartate aminotransferase, US, ultrasound, *Age > 50 years, diabetes, metabolic syndrome, increased alanine aminotransferase. 
progression to NASH and advanced fibrosis is uncommon for simple steatosis. ${ }^{103,104}$ However, irrespective of the stage of fibrosis, considering the increased risk of metabolic syndrome and diabetes, laboratory parameters for metabolic tests should be repeated every 6-12 months. Patients more than 45 years of age should also undergo periodic evaluation for cardiovascular risk.

A liver biopsy is usually done for monitoring of NAFLD progression in clinical trials. A reduction of fibrosis stage of 1 or more is used as a criteria for disease regression. However, it is unrealistic to perform a liver biopsy for the monitoring of fibrosis progression in clinical practice. Non-invasive methods using serum markers and/or TE as enumerated above may be more practical and they can be done repeatedly. Patients with no/mild fibrosis should be advised retesting for advanced liver fibrosis every 3 years for adults and every 2 years for children and young people using non-invasive methods. ${ }^{41}$

Patients with cirrhosis should be screened for varices and $\mathrm{HCC}$ as per standard guidelines.

\section{Conclusions}

NAFLD is a common disorder and can cause significant liver disease. NAFLD is associated with metabolic syndrome, type 2 diabetes mellitus and increased cardiovascular risk. For appropriately managing patients with NAFLD, it is important to assess the severity of the disease which includes differentiating between NAFL and NASH, quantifying the amount of fibrosis and identification of patients with cirrhosis and portal hypertension. Various non-invasive methods are available for assessment of disease severity and monitoring of disease progression. Weight loss measures by diet and exercise remain the mainstay of management and drug therapy and should be considered for patients with advanced fibrosis, high necro-inflammation or in patients with early stage NASH, if they are at a high risk of disease progression (age $>50$ years, diabetes mellitus, metabolic syndrome, increased alanine aminotransferase levels).

\section{References}

1. Caldwell S, Argo C. The natural history of non-alcoholic fatty liver disease. Dig Dis. 2010;28(1):162-8.
2. Brunt EM, Neuschwander-Tetri BA, Burt AD. Fatty liver disease: Alcoholic and non-alcoholic. In: Burt AD, Portmann B, Ferrel L (Eds.). MacSween's Pathology of the Liver. Edinburgh: Churchill Livingstone. 2012:293-359.

3. Burt DA, Lackner C, Tiniakos DG. Diagnosis and assessment of NAFLD: Definitions and histopathological classification. Semin Liver Dis. 2015;35:207-20.

4. Schneier AT, Citti CC, Dieterich DT. Management and diagnosis of fatty liver disease Expert Rev. Gastroenterol Hepatol. 2015;9(5):671-83.

5. Review Team, LaBrecque DR, Abbas Z, et al. World Gastroenterology Organisation global guidelines: Nonalcoholic fatty liver disease and nonalcoholic steatohepatitis. J Clin Gastroenterol. 2014;48:467-73.

6. Clark JM, Diehl AM. Defining nonalcoholic fatty liver disease: implications for epidemiologic studies. Gastroenterology. 2003;124:248-50.

7. Browning JD, Szczepaniak LS, Dobbins R, et al. Prevalence of hepatic steatosis in an urban population in the United States: impact of ethnicity. Hepatology. 2004;40:1387-95.

8. Bellentani S, Saccoccio G, Masutti F, et al. Prevalence of and risk factors for hepatic steatosis in Northern Italy. Ann Intern Med. 2000;132:112-7.

9. Fan JG, Zhu J, Li XJ, et al. Prevalence of and risk factors for fatty liver in a general population of Shanghai, China. J Hepatol. 2005;43:508-14.

10. Duseja A, Singh SP, Saraswat VA, et al. Non-alcoholic Fatty Liver Disease and Metabolic Syndrome Position Paper of the Indian National Association for the Study of the Liver, Endocrine Society of India, Indian College of Cardiology and Indian Society of Gastroenterology. J Clin Exp Hepatol. 2015;5:51-68.

11. Duseja A, Chawla Y. Nonalcoholic fatty liver disease in India - how much? How soon? Trop Gastroenterol. 2005; 26:1-3.

12. Byrne CD, Targher G. NAFLD: a multisystem disease. J Hepatol. 2015;62:S47-S64.

13. Gastaldelli A, Kozakova M, Højlund K, et al. Fatty liver is associated with insulin resistance, risk of coronary heart disease, and early atherosclerosis in a large European population. Hepatology. 2009;49:1537-44.

14. Ekstedt M, Franzén LE, Mathiesen UL, et al. Long-term follow-up of patients with NAFLD and elevated liver enzymes. Hepatology. 2006;44:865-73.

15. Day CP, James OF. Steatohepatitis: a tale of two "hits"? Gastroenterology. 1998;114(4):842-5.

16. Cortez-Pinto H, de Moura M, Day C. Non-alcoholic 
steatohepatitis: from cell biology to clinical practice. J Hepatol. 2006;44:197-208.

17. Buzzetti E, Pinzani M, Tsochatzis EA. The multiple-hit pathogenesis of non-alcoholic fatty liver disease (NAFLD). Metabol Clin Exp. 2016;65:1038-48.

18. Herbert Tilg, Alexander R. Moschen. Evolution of Inflammation in Nonalcoholic Fatty Liver Disease: The Multiple Parallel Hits Hypothesis; Hepatology. 2010;52:1836-46.

19. Donnelly KL, Smith CI, Schwarzenberg SJ, et al. Sources of fatty acids stored in liver and secreted via lipoproteins in patients with nonalcoholic fatty liver disease. J Clin Invest. 2005;115:1343-51.

20. Machado MV, Diehl AM. Pathogenesis of Nonalcoholic Steatohepatitis. Gastroenterology. 2016;150:1769-77.

21. Matteoni CA, Younossi ZM, Gramlich T, et al. Non alcoholic fatty liver disease: a spectrum of clinical and pathological severity. Gastroenterology. 1999;116:1413-9.

22. Angulo P. Long-term mortality in nonalcoholic fatty liver disease: is liver histology of any prognostic significance? Hepatology. 2010;51(2):373-5.

23. Argo CK, Caldwell SH. Epidemiology and natural history of non-alcoholic steatohepatitis. Clin Liver Dis. 2009;13:511-31.

24. Pais R, Pascale A, Fedchuck L, Charlotte F, Poynard T, Ratziu V. Progression from isolated steatosis to steatohepatitis and fibrosis in nonalcoholic fatty liver disease. Clin Res Hepatol Gastroenterol. 2011;35:23-8.

25. Yilmaz Y. Review article: is non-alcoholic fatty liver disease a spectrum, or are steatosis and non-alcoholic steatohepatitis distinct conditions? Aliment Pharmacol Ther. 2012;36:815-23.

26. Singh S, Allen AM, Wang Z, Prokop LJ, Murad MH, Loomba R. Fibrosis progression in nonalcoholic fatty liver vs nonalcoholic steatohepatitis: a systematic review and meta-analysis of paired-biopsy studies. Clin Gastroenterol Hepatol. 2015;13:643-54, e641-e649; quiz e639-40.

27. Chalasani N, Younossi Z, Lavine JE, et al. The diagnosis and management of non-alcoholic fatty liver disease: practice guideline by the American Gastroenterological Association, American Association for the Study of Liver Diseases, and American College of Gastroenterology. Gastroenterology. 2012;142:1592-609.

28. Adams LA, Talwalkar JA. Diagnostic evaluation of non-alcoholic fatty liver disease. J Clin Gastroenterol. 2006; 40:S34-8.

29. Loguercio C, De Simone T, D'Auria MV, et al. Non- alcoholic fatty liver disease: a multicentre clinical study by the Italian Association for the Study of the Liver. Dig Liver Dis. 2004;36:398-405.

30. Setji TL, Holland ND, Sanders LL, et al. Nonalcoholic steatohepatitis and non-alcoholic fatty liver disease in young women with polycystic ovary syndrome. J Clin Endocrinol Metab. 2006;91:1741-7.

31. National Institutes of Health: Third Report of the National Cholesterol Education Program Expert Panel on Detection, Evaluation, and Treatment of High Blood Cholesterol in Adults (Adult Treatment Panel III) Executive Summary. Bethesda, MD, National Institutes of Health, National Heart, Lung and Blood Institute. 2001 (NIH publ. no. 013670)

32. Misra A, Chowbey PK, Makkar BM, et al. Consensus statement for diagnosis of obesity, abdominal obesity and the metabolic syndrome for Asian Indians and recommendations for physical activity, medical and surgical management. J Assoc Physicians India. 2009;57:163-70.

33. Saadeh S, Younossi ZM, Remer EM, et al. The utility of radiological imaging in nonalcoholic fatty liver disease. Gastroenterology. 2002;123:745-50.

34. Lee SS, Park SH, Kim HJ, Kim SY, Kim MY, Kim DY, Suh DJ, Kim KM, Bae MH, Lee JY, Lee SG, Yu ES. Non-invasive assessment of hepatic steatosis: prospective comparison of the accuracy of imaging examinations. J Hepatol. 2010;52:579-85.

35. Lee SS, Park SH. Radiological evaluation of nonalcoholic fatty liver disease. World J Gastroenterol. 2014;20(23): 7392-402.

36. Angulo P, Hui JM, Marchesini G, et al. The NAFLD fibrosis score: a noninvasive system that identifies liver fibrosis in patients with NAFLD. Hepatology. 2007;45:846-54.

37. Kwok R, Tse YK, Wong GL, Ha Y, Lee AU, Ngu MC, Chan HL, Wong VW. Systematic review with metaanalysis: non-invasive assessment of non-alcoholic fatty liver disease- the role of transient elastography and plasma cytokeratin-18 fragments. Aliment Pharmacol Ther. 2014;39:254-69.

38. Sterling RK, Lissen E, Clumeck N, et al. Development of a simple noninvasive index to predict significant fibrosis in patients with $\mathrm{HIV} / \mathrm{HCV}$ coinfection. Hepatology. 2006;43:1317-25.

39. Ratziu V, Massard J, Charlotte F, et al. Diagnostic value of biochemical markers (Fibro Test-FibroSURE) for the prediction of liver fibrosis in patients with non-alcoholic fatty liver disease. BMC Gastroenterol. 2006; 6:6. 
40. Rosenberg WM, Voelker M, Thiel R, et al. Serum markers detect the presence of liver fibrosis: a cohort study. Gastroenterology. 2004;127:1704-13.

41. National Institute of Health and Care Excellence (NICE). Non-alcoholic fatty liver disease (NAFLD): assessment and management. NICE guideline [NG49] Published date: July 2016. Available at: https://www.nice.org.uk/guidance/ ng49? unlid=425264848201610484926.

42. Wang Y, Fan Q, Wang $T$ et al. Controlled attenuation parameter for assessment of hepatic steatosis grades: a diagnostic meta-analysis. Int $J$ Clin Exp Med. 2015;8(10):17654-63.

43. Castera L, Foucher J, Bernard PH, et al. Pitfalls of liver stiffness measurement: a 5-year prospective study of 13,369 examinations. Hepatology. 2010;51:828-35.

44. Myers RP, Pomier-Layrargues G, Kirsch R, et al. Discordance in fibrosis staging between liver biopsy and transient elastography using the fibroscan $\mathrm{xl}$ probe. J Hepatol. 2011;56:564-70.

45. Bedossa P, Patel K. Biopsy and non-invasive methods to assess progression of nonalcoholic fatty liver disease. Gastroenterology. 2016;150(8):1811-22.

46. European Association for the Study of the Liver (EASL), European Association for the Study of Diabetes (EASD) and European Association for the Study of Obesity (EASO). EASL-EASD-EASO Clinical Practice Guidelines for the management of non-alcoholic fatty liver disease. J Hepatol. 2016;64:1388-402.

47. Zhu JZ, Hollis-Hansen K, Wan XY, et al. Clinical guidelines of non-alcoholic fatty liver disease: A systematic review. World J Gastroenterol. 2016;22(36):8226-33.

48. Musso G, Cassader M, Rosina F, Gambino R. Impact of current treatments on liver disease, glucose metabolism and cardiovascular risk in non-alcoholic fatty liver disease (NAFLD): a systematic review and meta-analysis of randomised trials. Diabetologia. 2012;55:885-904.

49. Promrat K, Kleiner DE, Niemeier HM, et al. Randomized controlled trial testing the effects of weight loss on nonalcoholic steatohepatitis. Hepatology. 2010;51:121-9.

50. Vilar-Gomez E, Martinez-Perez Y, Calzadilla-Bertot L, et al. Weight loss via lifestyle modification significantly reduces features of nonalcoholic steatohepatitis. Gastroenterology. 2015;149:367-78.

51. Glass LM, Dickson RC, Anderson JC, et al. Total body weight loss of $\geq 10 \%$ is associated with improved hepatic fibrosis in patients with nonalcoholic steatohepatitis. Dig Dis Sci. 2015;60:1024-30.
52. Hannah Jr. WN, Harrison SA. Lifestyle and Dietary Interventions in the Management of Nonalcoholic Fatty Liver Disease. Dig Dis Sci. 2016;61(5):1365-74.

53. Nguyen V, George J. Nonalcoholic Fatty Liver Disease Management: Dietary and Lifestyle Modifications. Semin Liver Dis. 2015;35:318-37.

54. Oliveira CP, de Lima SP, de Abreu-Silva EO, Marcadenti A. Nutrition and physical activity in nonalcoholic fatty liver disease. J Diabetes Res. 2016; 2016: 4597246. http:// dx.doi.org/10.1155/2016/4597246.

55. Mahady SE, George J. Exercise and diet in the management of nonalcoholic fatty liver disease. Metabolism. 2015;65:1172-82.

56. Shai I, Schwarzfuchs D, Henkin Y, et al; Dietary Intervention Randomized Controlled Trial (DIRECT) Group. Weight loss with a low-carbohydrate, Mediterranean, or low-fat diet. N Engl J Med. 2008;359(3):229-41.

57. Lassailly G, Caiazzo R, Pattou F, Mathurin P. Perspectives on treatment for nonalcoholic steatohepatitis. Hastroenterology. 2016;150:1835-48.

58. Davies MJ, Bergenstal R, Bode B, et al. Efficacy of liraglutide for weight loss among patients with type 2 diabetes: the SCALE diabetes randomized clinical trial. JAMA. 2015;314:687-99.

59. Astrup A, Rossner S, Van Gaal L, et al. Effects of liraglutide in the treatment of obesity: a randomised, double-blind, placebo-controlled study. Lancet. 2009;374:1606-16.

60. Sullivan S, Kirk EP, Mittendorfer B, Patterson BW, Klein S. Randomized trial of exercise effect on intrahepatic triglyceride content and lipid kinetics in nonalcoholic fatty liver disease. Hepatology. 2012;55(6):1738-45.

61. Oh S, Shida T, Yamagishi K, et al. Moderate to vigorous physical activity volume is an important factor for managing nonalcoholic fatty liver disease: a retrospective study. Hepatology. 2015;61:1205-15.

62. Zelber-Sagi S, Buch A, Yeshua H, et al. Effect of resistance training on non-alcoholic fatty-liver disease a randomizedclinical trial. World J Gastroenterol. 2014;20(15):4382-92.

63. Hallsworth K, Fattakhova G, Hollingsworth KG, et al. Resistance exercise reduces liver fat and its mediators in non-alcoholic fatty liver disease independent of weight loss. Gut. 2011;60(9):1278-83.

64. Mummadi RR, Kasturi KS, Chennareddygari S, Sood GK. Effect of bariatric surgery on nonalcoholic fatty liver disease: systematic review and meta-analysis. Clin Gastroenterol Hepatol. 2008;6(12):1396-402.

65. Lassailly G, Caiazzo R, Pattou F, et al. Bariatric surgery 
for curing NASH in the morbidly obese? $J$ Hepatol. 2013;58:1249-51.

66. Caiazzo R, Lassailly G, Leteurtre E, et al. Roux-en-Y gastric bypass versus adjustable gastric banding to reduce nonalcoholic fatty liver disease: a 5-year controlled longitudinal study. Ann Surg. 2014;260:893-9.

67. Chavez-Tapia NC, Tellez-Avila FI, Barrientos-Gutierrez T, Mendez-Sanchez N, Lizardi-Cervera J, Uribe M. Bariatric surgery for non-alcoholic steatohepatitis in obese patients. Cochrane Database Syst Rev. 2010;(1):CD007340.

68. Rouabhia S, Milic N, Abenavoli L. Metformin in the treatment of non-alcoholic fatty liver disease: Safety, efficacy and mechanism. Expert Rev Gastroenterol Hepatol. 2014;8(4):343-9.

69. Loomba R, Lutchman G, Kleiner DE, et al. Clinical trial: pilot study of metformin for the treatment of non-alcoholic steatohepatitis. . 2009;29:172-82.

70. Marchesini G, Brizi M, Bianchi G, et al. Metformin in nonalcoholic steatohepatitis. Lancet. 2001;358:893-94.

71. Bugianesi E, Gentilcore E, Manini R, et al. A randomized controlled trial of metformin versus vitamin $\mathrm{E}$ or prescriptive diet in nonalcoholic fatty liver disease. $\mathrm{Am} \mathrm{J}$ Gastroenterol. 2005;100:1082-90.

72. Rakoski MO, Singal AG, Rogers MA, et al. Meta-analysis: insulin sensitizers for the treatment of non-alcoholic steatohepatitis. Aliment Pharmacol Ther. 2010;32:121121.

73. Zhang ZJ, Zheng ZJ, Shi R, Su Q, Jiang Q, Kip KE. Metformin for liver cancer prevention in patients with type 2 diabetes: a systematic review and metaanalysis. J Clin Endocrinol Metab. 2012;97:2347-53.

74. Chen HP, Shieh JJ, Chang CC, et al. Metformin decreases hepatocellular carcinoma risk in a dose-dependent manner: population-based and in vitro studies. Gut. 2012;62:60615.

75. Ballestri S, Nascimbeni F, Romagnoli D, Baldelli E, Lonardo A. The role of nuclear receptors in the pathophysiology, natural course, and drug treatment of NAFLD in humans. Adv Ther. 2016;33(3):291-319.

76. Sanyal AJ, Chalasani N, Kowdley KV, et al. Pioglitazone, vitamin $\mathrm{E}$, or placebo for nonalcoholic steatohepatitis. N Engl J Med. 2010;362:1675-85.

77. Belfort R, Harrison SA, Brown K, et al. A placebocontrolled trial of pioglitazone in subjects with nonalcoholic steatohepatitis. N Engl J Med. 2006;355:2297-307.

78. Promrat K, Lutchman G, Uwaifo GI, et al. A pilot study of pioglitazone treatment for nonalcoholic steatohepatitis.
Hepatology. 2004;39:188-96.

79. Aithal GP, Thomas JA, Kaye PV, etal. Randomized, placebocontrolled trial of pioglitazone in nondiabetic subjects with nonalcoholic steatohepatitis. Gastroenterology. 2008;135: 1176-84.

80. Boettcher E, Csako G, Pucino F, Wesley R, Loomba R. Metaanalysis: pioglitazone improves liver histology and fibrosis in patients with non-alcoholic steatohepatitis. Aliment Pharmacol Ther. 2012;35(1):66-75.

81. Bennett WL, Maruthur NM, Singh S, et al. Comparative effectiveness and safety of medications for type 2 diabetes: an update including new drugs and 2-drug combinations. Ann Intern Med. 2011;154(9):602-13.

82. Neumann A, Weill A, Ricordeau P, et al. Pioglitazone and risk of bladder cancer among diabetic patients in France: a population-based cohort study. Diabetologia. 2012;55:1953-62

83. Azoulay L, Yin H, Filion KB, et al. The use of pioglitazone and the risk of bladder cancer in people with type 2 diabetes: nested case-control study. BMJ. 2012;344:e3645

84. Ratziu V, Harrison S, Francque S, et al. Elafibranor, an agonist of the peroxisome proliferator-activated receptor- $\alpha$ and $-\delta$, induces resolution of nonalcoholic steatohepatitis without fibrosis worsening. Gastroenterology. 2016;150:1147-59.

85. Rotman Y, Sanyal AJ. Current and upcoming pharmacotherapy for non-alcoholic fatty liver disease. Gut. 2016;19. pii: gutjnl-2016-312431. doi: 10.1136/ gutjnl-2016-312431. [Epub ahead of print]

86. Neuschwander-Tetri BA, Loomba R, SanyalAJ,et al; NASH clinical research network. Farnesoid X nuclear receptor ligand obeticholic acid for non-cirrhotic, non-alcoholic steatohepatitis (FLINT): A multicentre, randomised, placebo-controlled trial. Lancet. 2015;385(9972):956-65.

87. Abu-Hamdah R, Raibee A, Meneilly GS, et al. Clinical review: The extrapancreatic effects of glucagon-like peptide-1 and related peptides. J Clin Endocrinol Metab. 2009;94:1843-52.

88. Armstrong MJ, Gaunt P, Aithal GP, et al. Liraglutide safety and efficacy in patients with non-alcoholic steatohepatitis (LEAN): a multicenter, double-blind randomized, placebocontrolled phase 2 study. Lancet. 2016;387:679-90.

89. Lavine JE, Schwimmer JB, Van Natta ML, et al. Effect of vitamin $\mathrm{E}$ or metformin for treatment of nonalcoholic fatty liver disease in children and adoloscents: the TONIC randomized controlled trial. JAMA. 2011;305:1659-68.

90. Schurks M, Glynn RJ, Rist PM, et al. Effects of vitamin E 
on stroke subtypes: meta-analysis of randomised controlled trials. BMJ. 2010;341:c5702.

91. Klein EA, Thompson IM Jr, Tangen CM, et al. Vitamin $\mathrm{E}$ and the risk of prostate cancer: the Selenium and Vitamin E Cancer Prevention Trial (SELECT). JAMA. 2011;306:1549-56.

92. Miller ER 3rd, Pastor-Barriuso R, Dalal D, et al. Metaanalysis: high-dosage vitamin E supplementation may increase all-cause mortality. Ann Intern Med. 2005;142:3746.

93. Du J, Ma YY, Yu CH, et al. Effects of pentoxifylline on nonalcoholic fatty liver disease: a meta-analysis. World $J$ Gastroenterol. 2014;20:569-77.

94. Scorletti E, Bhatia L, McCormick KG, et al. Effects of purified eicosapentaenoic and docosahexaenoic acids in nonalcoholic fatty liver disease: results from the Welcome study. Hepatology. 2014;60:1211-21.

95. Sanyal AJ, Abdelmalek MF, Suzuki A, et al. No significant effects of ethyl-eicosapentanoic acid on histologic features of nonalcoholic steatohepatitis in a phase 2 trial. Gastroenterology. 2014;147:377-384 e1.

96. Kargiotis K, Athyros VG, Giouleme O, et al. Resolution of non-alcoholic steatohepatitis by rosuvastatin monotherapy in patients with metabolic syndrome. World J Gastroenterol. 2015;21(25):7860-8.

97. Oza N, Takahashi H, Eguchi Y, et al. Efficacy of ezetimibe for reducing serum low-density lipoprotein cholesterol levels resistant to lifestyle intervention in patients with non-alcoholic fatty liver disease. Hepatol Res. 2014;44(7): 812-7.
98. Takeshita Y, Takamura T, Honda M, et al. The effects of ezetimibe on non-alcoholic fatty liver disease and glucose metabolism: A randomised controlled trial. Diabetologia. 2014;57(5):878-90.

99. Loomba R, Sirlin CB, Ang B, et al. Ezetimibe for the treatment of nonalcoholic steatohepatitis: assessment by novel magnetic resonance imaging and magnetic resonance elastography in a randomized trial (MOZART trial). Hepatology. 2015;61:1239-50.

100. Filozof C, Goldstein BJ, Williams RN, Sanyal A. NonAlcoholic Steatohepatitis: Limited Available Treatment Options but Promising Drugs in Development and Recent Progress Towards a Regulatory Approval Pathway. Drugs. 2015;75(12):1373-92.

101.Newsome PN, Allison ME, Andrews PA, et al. Guidelines for liver transplantation for patients with non-alcoholic steatohepatitis. Gut. 2012;61:484-500.

102.D, Kim WR, Kim HJ, Therneau TM. Association between noninvasive fibrosis markers and mortality among adults with nonalcoholic fatty liver disease in the United States. Hepatology. 2013;57:1357-65.

103.Pais R, Charlotte F, Fedchuk L, et al. A systematic review of follow-up biopsies reveals disease progression in patients with non-alcoholic fatty liver. J Hepatol. 2013;59:550-6.

104. McPherson S, Hardy T, Henderson E, Burt AD, Day $\mathrm{CP}$, Anstee QM. Evidence of NAFLD progression from steatosis to fibrosing-steatohepatitis using paired biopsies: implications for prognosis and clinical management. J Hepatol. 2015;62:1148-55. 